Research Paper

\title{
Mapping and characterizing the Jefoure roads that have cultural heritage values in the Gurage socio-ecological production landscape of Ethiopia
}

\author{
M. Sahle ${ }^{a, b, *, 1}$, O. Saito ${ }^{c, 2}$ \\ ${ }^{\text {a }}$ United Nations University Institute for the Advanced Study of Sustainability, Tokyo, Japan \\ ${ }^{\mathrm{b}}$ Department of Natural Resources Management, Wolkite University, Wolkite, Ethiopia \\ ${ }^{\mathrm{c}}$ Institute for Global Environmental Strategies, Kanagawa, Japan
}

\section{H I G H L I G H T S}

- The Gurage people in Ethiopia designs a unique grass-cover road called Jefoure.

- Jefoure roads have long lengths and wide breadth, but they are not consistent.

- The physical geography of the landscape has an impact on the Jefoure roads.

- Jefoure roads are a multifunctional road.

- The Jefoure roads and their evolving landscape require international recognition.

\section{A R T I C L E I N F O}

\section{Keywords:}

Landscape characterization

Cultural landscape

Gurage people

Physical geography

Multifunctional road

Heritagization

\begin{abstract}
A B S T R A C T
Information about cultural landscape settings and characteristics is essential for understanding and finding solutions for their sustainable management. In the Gurage socio-ecological production landscape of Ethiopia, "Jefoure" refers to a traditional grass-covered road with households on either side. This study aims to map and characterize Jefoure roads to help manage them sustainably. Data were compiled using survey tools and recent orthophoto images and then were qualitatively and quantitatively analyzed. The results underscore Jefoure as a network of roads of variable lengths that can be as extensive as $87 \mathrm{~m}$. Notably, the physical geography of the landscape has an impact on the length, shape, and directions of these roads. Jefoure roads are multifunctional roads that have been designed and managed by local people for centuries. The Gurage settlement pattern is centered on these roads, which influences their home garden functional spaces. Residents, local groups, land-use planners, and decision-makers need to manage the cultural roads sustainably. This study generates valuable empirical information regarding a Gurage landscape feature that is not common knowledge, and it can support decision-makers and other conservation initiatives aimed at the sustainable management. It may also inform and encourage other researchers in their studies on similar cultural roads. This road has a cultural value and needs to work on its heritagization, in conjunction with the community's various cultural assets.
\end{abstract}

\section{Introduction}

Socio-ecological production landscapes are characterized by a mosaic of ecosystems such as secondary forests, home garden agroforestry, grasslands, water bodies, and human settlement. They are managed through interactions between ecosystems and humans to create various goods and services for human well-being (Japan
Satoyama Satoumi Assessment, 2010; Takeuchi, 2010). The term "cultural landscapes" is often used synonymously for similar landscapes where people have developed and sustainably managed the landscape over a long time (Saito, Subramanian, Hashimoto, \& Takeuchi, 2020). The World Heritage Committee states that cultural landscapes are produced by long-term interactions between people and nature within indigenous societies (United Nations Educational, Scientific and

\footnotetext{
* Corresponding author at: United Nations University Institute for the Advanced Study of Sustainability, Tokyo, Japan.

E-mail addresses: achemo@unu.edu, mesitago@gmail.com (M. Sahle), o-saito@iges.or.jp (O. Saito).

1 https://orcid.org/0000-0002-2280-9957.

2 https://orcid.org/0000-0002-0697-9593.
} 
Cultural Organization [UNESCO], 2015). They represent the continuous modification of the natural landscape by indigenous people as a means to better adapt land uses and spatial structures to meet changing human demands (Antrop, 2005; Campolo, Bombino, \& Meduri, 2016; Fisher, Turner, \& Morling, 2009; Huntsinger \& Oviedo, 2014). These landscapes are a vital part of daily living environments and include scenery with aesthetic and recreational qualities (Hartel et al., 2014), feature biodiversity (Herzog, 1998), and provide goods and services to society (Plieninger, van der Horst, Schleyer, \& Bieling, 2014). Cultural landscapes reflect specific practices of sustainable land-use (UNESCO, 2015) and can be multifunctional (Rabbinge \& Bindraban, 2012). Cultural roads and routes are characteristics of cultural landscapes (Brenna, Larsen, \& Hvattum, 2011). Roads shape and reshape valuable and commonplace landscapes, enhancing and/or diminishing their qualities. Over time, roads and their elements can become cultural heritage objects themselves because they represent technical or historical phases of cultural landscape development and interconnections with works of art (Grazuleviciute-Vileniske \& Matijosaitiene, 2010).

Cultural landscapes and their road development are undergoing rapid and fundamental transformations, chiefly because of sustainable management challenges linked to urban and rural land-use (Verburg, van Berkel, van Doorn, van Eupen, \& van den Heiligenberg Harm, 2010). Cultural landscape changes are driven by changes to institutional arrangements, demographics, economic conditions, infrastructure provisions, climate, and policies (Plieninger et al., 2013). These changes have resulted in standardized and mechanized land-use methods gradually replacing traditional landscape practices (Kizos \& Vlahos, 2012). These changes can lead to the deprivation of landscape assets, leaving the future of many cultural landscapes highly uncertain (Plieninger et al., 2014).

Accordingly, understanding, retaining, developing, and sustainably using traditional landscapes are increasingly considered important topics in discussions among experts on geography, regional planning, landscape planning, and cultural heritage conservation (Rotondo, 2016). In recent years, several initiatives have called for integrated landscape approaches within socio-ecological production landscape management. For example, the Satoyama Initiative (Takeuchi, 2010) is among the internationally recognized initiatives that foster the management and sustainable use of biodiversity and ecosystem services in human-influenced landscapes. These initiatives aim to preserve regional diversity and the heritage of cultural landscapes while identifying pathways to a more sustainable future (Plieninger et al., 2014).

Rural cultural landscapes, which can be understood as coupled social-ecological systems, support human well-being and development (Parrott \& Meyer, 2012). Large portions of the African continent's people live in rural landscapes close to nature. Several African cultural landscapes that were developed by long-term interactions with nature are listed as World Heritage sites (African World Heritage, 2018). Various groups of Ethiopian people developed diverse cultural values based on their indigenous knowledge; these include the Konso and Gedio cultural landscapes (UNU-IAS \& IR3S/UTIAS, 2016; Watson, 2009). The Gurage socio-ecological production landscape in Ethiopia is characterized by a mosaic of different ecosystem types such as forests, home garden agroforestry system, cereal crops, grasslands, woodlots, wetlands, surface water, and roads, as well as human settlements. The Gurage people are a group in Ethiopia that formed village settlements based on the ecological conditions linked to Jefoure roads and Enset culture (Shack, 1966).

Jefoure roads are long and wide grass-covered streets that run through the middle of Gurage villages. Houses and trees flank these roads on both sides. The roads connect villages and have long served as a medium of transport to main roads, markets, and towns within the Gurage socio-ecological production landscape of Ethiopia. In Western Gurage, the establishment of a formal road network began in the 1960s (Nida, 2000). However, road networks in the region remain limited (GZFED, 2018). The bulk of rural people use Jefoure green roads as a medium of transport on foot, the backs of animals, and in motorized vehicles in large landscape areas. Additionally, the roads present open spaces running through villages and serve as public gathering areas, mourning areas, squares for making bonfires, wedding venues, communal grazing sites, and playgrounds for children and youths (Yirga, Abera, Kebede, \& Kifle, 2012).

Although the Gurage socio-ecological production landscape has an exemplary rural landscape and road design comparable to modern urban planning and road networks, we could not find any well-documented study on the landscape characteristics. The roads' spatial dimensions, biophysical characteristics, hierarchies within roads, management practices, socio-cultural and environmental contributions, and heritage values have to date not been studied in detail. Accordingly, there is an increasing demand on society to better understand the road designs and the general landscape characteristics to enable their conservation, sustainable management, and promotion.

Landscape characterization approaches demonstrate the distinct features and values that the current environment can generate (Warnock \& Griffiths, 2014). By engaging with these landscape characterizations, unified communication in management and research can be achieved (Hazeu et al., 2011; Simensen, Halvorsena, \& Erikstad, 2018). Mapping and characterizing the Gurage socio-ecological production landscape with its integral Jefoure roads can assist for documentation and communication; enhancing the understanding of residents, land-use planners, and decision-makers; and providing support for conservation and the development of sustainable management. This paper aims to map and characterize Jefoure cultural roads and their surrounding environments to enable their sustainable management in the Gurage cultural landscape of Ethiopia. Since Jefoure roads influence settlement patterns and the existing research on Jefoure roads is lacking, our focus is on the roads themselves. The generated data can also assist work linked to the roads' heritage value as it concerns the Gurage sociocultural landscape's various traditional characteristics. The literature indicates that previous studies on-road characteristics have primarily focused on paved roads within rural and urban areas, heritage sites, and historical routes. However, studies exploring grass-covered roads that exist in socio-ecological production landscapes are lacking. The current research can thus help to enhance our understanding of indigenous grass-covered road designs and management.

This paper is organized into five sections. The second section describes the materials and methods used in this study and includes a description of the study area. The findings are presented in the third section and include six sub-sections that outline the overall landscape features and describe the spatial, physical geography, design trends, management, and socio-cultural characteristics of Jefoure roads. The major findings are discussed in section four. The fifth and final section concludes the study.

\section{Materials and methods}

\subsection{Study area}

The Gurage people inhabit a semi-mountainous region in southcentral Ethiopia, approximately $155 \mathrm{~km}$ southwest of the capital, Addis Ababa (Fig. 1). The Gurage socio-ecological production landscape is bordered by the Awash River basin to the north, the Gibe River (a large tributary of the Omo-Gibe basin) to the southwest, the Rift Valley basin to the east, and the Bilate River catchment to the south. Currently, the Gurage zone is under the Southern Nation, Nationalities, and Peoples' Region administration, while the northern, western, and eastern portions share a border with the Oromia Regional State. The Gurage zone has an approximate area size of $5932 \mathrm{~km}^{2}$. The zone has 13 woredas (districts) (Fig. 1). Based on the 2007 census conducted by the Central statistical Agency (CSA) (CSA) (2009) of Ethiopia, the Gurage zone has a total population of $1,279,646$ ( 622,078 men and 657,568 women). The overwhelming majority of the population $(92.4 \%)$ lives in rural areas 


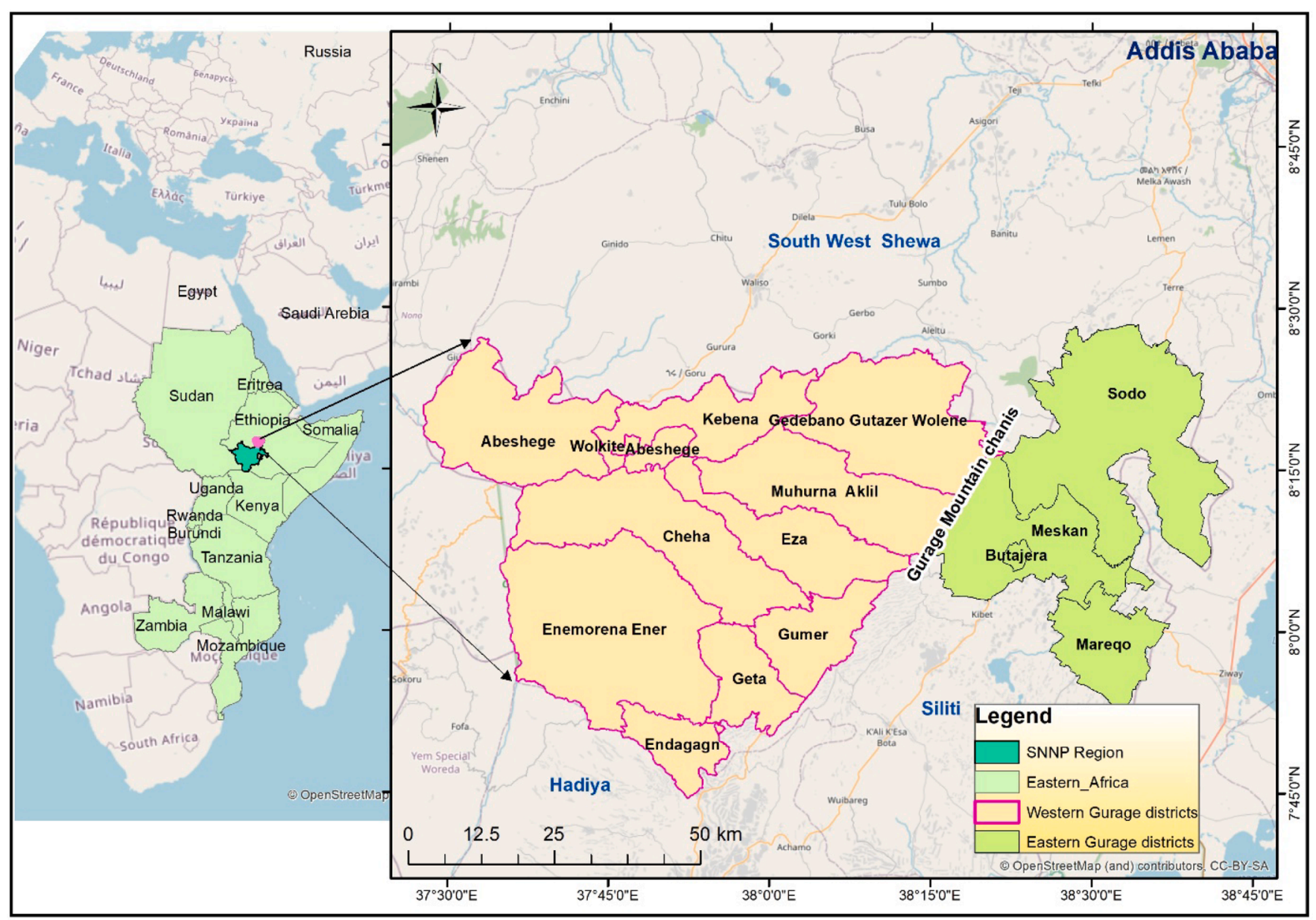

Fig. 1. Map of Western Gurage in Ethiopia.

and lead a subsistence life involving a mixed crop rotation system, transplanting, and livestock rearing.

In the Gurage landscape area, Cushitic and Semitic-speaking groups have settled and lived for nearly a millennium alongside the native inhabitants (Tadesse, 2009; Zewde, 1972). Various settlers arrived at different periods, bringing their own religious and cultural backgrounds with them. They assimilated with the local people and developed diverse tribes and languages/dialects (Hudson, 1996; Tadesse, 2009). Although the Gurage people adopt various languages/dialects and religions, they share a similar set of artifacts, technologies, modes of production, housebuilding designs, settlement patterns, and economic and social organization (Shack, 1966). They adapted Enset (Ensete ventricosum) crop cultivation mechanisms, producing the root crop in abundance as an indigenous staple/co-staple harvest in the region (Sahle, Yeshitela, \& Saito, 2018; Shack, 1966). The Enset crop is one of the primary home garden food crops in Ethiopia and resembles a large, thick, singlestemmed banana plant (Borrell et al., 2019; Brandt et al., 1997).

As a traditional system, Jefoure roads exist in different parts of the Gurage zone including Eastern Gurage (Fig. 1). The zone surrounding Gurage, which includes Siliti, Hadiya, and Southwest Shewa, shares similar traditions. Jefoure road networks exist widely in Western Gurage and, as such, this study adopted these networks as its focus.

\subsection{Methods}

To generate relevant data for characterizing Jefoure roads, we considered landscape and specific site-level data. All Jefoure roads in the landscape were extracted from orthophoto images. For specifically selected sites, in-depth interviews, focus group discussions (FGDs), and
Jefoure tree recording was conducted during February and March 2020. The data collected using survey tools and geospatial were qualitatively and quantitatively analyzed to characterize the Jefoure road landscape in terms of spatial extent, physical geography, history, design, management, and socio-cultural roles.

\subsubsection{The history, design, management, and socio-cultural roles of Jefoure roads}

In-depth interviews, FGDs, and Jefoure tree recording were conducted to generate data on the history, design, management, and sociocultural roles of Jefoure roads. This included describing the overall character of the socio-ecological production landscape. Twenty-one sites/villages were selected using the multistage stratified and random sampling method (see Fig. 2 and Supplementary material 1). These roads are not uniform throughout the landscape, and differences in length, width, agro-ecological zones, settlement history, and management practices were considered criteria for identifying representative sites. First, we categorized the landscape into three categories, based on the length of the roads as short $(<3 \mathrm{~km})$, medium $(3-6 \mathrm{~km})$, and long $(>7 \mathrm{~km})$. In the second stage, the roads were classified based on their width as narrow $(<20 \mathrm{~m})$, medium $(20-28 \mathrm{~m})$, and wide $(>28 \mathrm{~m})$, using the average width set by elders $(28 \mathrm{~m})$. The landscape has cool-moist, tepid-humid, and warm agro-ecological zones (AEZ); accordingly, roads were re-categorized based on their existing AEZ zones. In the fourth stage, roads were reclassified into their respective administrative districts.

Differences in settlement histories (long-term and relatively new) and management practices were considered as additional criteria in consultation with zone-specific Tourism Culture and Sports offices to 




Fig. 2. Selected Jefoure roads on a Landsat false-color composite image of the Western Gurage landscape.

identify the study sites. An "upright" Jefoure road is known for having wide roads that continue long-distance in a straight shape, have extensive grass cover, good side fences, scattered trees, well-constructed traditional houses, villages of historical events occurred, and for having diversified home gardens. The offices helped us to include the known villages that incorporated the management practices reflecting these characteristics. These selection criteria led us to identify kebeles/subdistricts. Finally, we randomly selected one street in each locality, since most of them shared similar characteristics.

As a result of time constraints (COVID-19-related), we did not collect field data from the already selected eight Jefoure sites and considered them as geospatial data sites (Fig. 2). In these geospatial data sites, except for the personal interviews, FGDs, and observations, the remaining essential data were collected from orthophoto images. We assumed that the missed data would not have a significant impact because the collected field survey data from 13 sites integrated with all site's geospatial data would generate reasonable findings.

We conducted in-depth interviews with 26 selected key informants among households within the study landscape. Two individuals in every 13 villages were nominated by the communities based on their age, extensive knowledge about community norms and culture, Jefoure road principles, the functions and management of these roads, and understanding current problems within their respective localities (see Supplementary material 2). Thirteen FGDs were held with a group of five to nine persons in each (98 participants in total). During selection, the heterogeneity of individuals was also considered. The FGD participants were selected based on age ( $>40$ years old, $67 \%$ ), gender (female, $30 \%$ ), and community role (see Supplementary material 3). In both cases, elders and resource persons were considered for the survey. This was because the survey did not focus on the perceptions of the community but, rather, on gathering common knowledge about Jefoure roads and the landscape.

Several questions from the structured questionnaire were asked of the key informants and the FGD participants. The first of these questions related to the history of each locality. Some of the topics included in the interviews and discussions focused on land tenure, the aim of Jefoure roads, where settlement plans had been initiated, the responsible bodies for managing Jefoure roads, the role of trees on Jefoure, the side-fencing system, and previous and current cultural and social practices linked to Jefoure. Similar questions were asked of the key informants and the FGD participants. The key informant interviews aimed to collect information based on individual opinions and practices, while the FGD aimed to coherently synthesize information following individual interviews. Furthermore, the author observed general outlooks for Jefoure roads by participating in walk-throughs in different parts of the landscape supported by taking photographs.

A deductive approach was used to code qualitative data because we used guiding questions, which were developed based on previous experience of the landscape. All the responses collected from study sites were coded separately. The coding allowed us to classify all of the collected data. Comparisons between datasets and across responses were made, and sub-themes (e.g., general landscape character, historical extent, design assumptions, socio-cultural roles, and management of Jefoure roads) were developed. Across a group and in individual interviews, a common idea was considered for presenting the results. Except for some quantitative data, a narrative description approach was employed to characterize the history, design, management, and sociocultural roles of Jefoure roads; this included describing the overall character of the socio-ecological landscape.

Data about the name, type, and the number of trees on Jefoure roads 
and their fences were obtained from field recordings in the sampled villages. The existing fencing types and their height along Jefoure roads were determined during on-site visits, and by considering the average fencing type in a community at a given Jefoure road. For the eight sites in which field data were not collected, visual interpretation of orthophoto images and Google Earth Pro images were considered as an aid for counting the number of trees on Jefoure and for determining fencing types. The number of households along Jefoure roads was determined by counting the land parcels (obtained from district offices) and overlaying these on raw orthophoto images for verification.

\subsubsection{The spatial characteristics of Jefoure roads}

To understand the spatial characteristics of Jefoure roads, landscapelevel spatial data were generated from orthophoto images. The images obtained from the region's rural land administration office and have a spatial resolution of $0.15 \mathrm{~m}$. The orthophoto images were captured and orthorectified for land administration and certification purposes in 2017 and are of good quality for extracting Jefoure roads from other features within the landscape (Ethiopian Mapping Agency [EMA], 2017). We first considered common spectral-based classification approaches, e.g., supervised and unsupervised, as well as object-based classification to extract Jefoure roads from the diverse features within the landscape. However, we were unable to generate quality data in this regard because Jefoure roads are found in different AEZ, and the spectral reflectance values of Jefoure road-cover varied according to AEZ. An object-based approach was not feasible because Jefoure roads shapes could not easily be differentiated by digital methods of classification.

Despite requiring a significant amount of time, accurate data can be generated by visual interpretation using high-resolution images (Schepaschenko, See, \& Lesiv, 2019; Zanella, Sousa, Souza, Carvalho, \& Borém, 2012). As Jefoure roads can easily be identified from other landuse styles by understanding village settlement patterns, it is possible to extract Jefoure roads using high-resolution orthophoto images. We extracted all of the Jefoure roads' spatial features in the Western Gurage landscapes as a polygon using the ArcGIS 17 software package. In addition to orthophoto images, we used Google Earth Pro images as an aid to accurately extract Jefoure roads and to avoid including existing asphalt and gravel roads present in the landscape. During the field visit, additional ground verification was done to check the accuracy of the spatial data in the locations that could not be identified by visual interpretation. Ground verification at specific sites was conducted using printed orthophoto maps and global positioning system (GPS) data were employed to collect reference points. The boundaries of Jefoure roads were sketched on the maps and then scanned for digitization. Georeferencing was completed prior to digitization and the GPS referencing points were overlaid for further verification.

We used the extracted spatial data from the orthophoto images and calculated the length, width, and area of Jefoure roads for documentation and outlining management implications. We converted the polygon features to centerline using the Production Centerline tool in ArcGIS Pro to calculate each Jefoure road's length. Road length and width were reclassified into five classes for regrouping, and to observe the different ranges of Jefoure roads and the average differences between them. The XTools Pro 4.0 for ArcGIS Desktop was used to calculate the minimum, maximum, and average polygon width of Jefoure roads. For site-specific demonstration, we considered the data from the 21 sample sites. The generated data helped us to quantitatively describe Jefoure roads and to compare the average widths planned and assigned to Jefoure roads by local elders.

\subsubsection{Physical geographical characteristics of Jefoure roads}

The physical geographical characteristics of Jefoure roads were analyzed to explore the effects of topographic elements on them. The physical factors connected with the topography of the area, such as elevation and slope grade, were considered in this study. The elevation and slope grade data were derived from a digital elevation model with a resolution of $30 \mathrm{~m}$, obtained from United States Geological Survey Shuttle Radar Topography Mission satellite images. The spatial data were overlaid on elevation ranges and slope gradients to analyze their effects on the length, width, direction, shape, and beginning and ending points of Jefoure roads. These road features were categorized into diverse classes to conduct descriptive analyses. The generated output was anticipated to indicate how natural features affected Jefoure roads and helped to determine the landscape principles followed by the local communities when designing Jefoure roads.

\section{Results}

The following sub-sections describe Jefoure road characteristics according to categories as follows: the general character of the Gurage socio-ecological production landscape (Section 3.1); the historical trends and designs of Jefoure roads (Section 3.2); the spatial characteristics of Jefoure roads (Section 3.3); the physical geographical characteristics of Jefoure roads (Section 3.4); the socio-cultural and ecological characteristics of Jefoure roads (Section 3.5); the management of Jefoure roads (Section 3.6).

\subsection{The Gurage socio-ecological production landscape characteristics related to Jefoure roads}

Jefoure roads are streets in the middle of the Gurage socio-ecological production landscape that is flanked by houses and farmlands on either side (Fig. 3). Jefoure is covered with natural grasses and plays a significant role in governing the Gurage peoples' village settlements. Farmers settle on both the right and left sides of these roads. For the Jefoure roads included in this case study, the number of households ranged from 64 in Sefato to 555 in Atazo (see Supplemental material 4). On average, $36.8 \pm 13$ households settled on both sides of a $1 \mathrm{~km}$ Jefoure road. Each household had one, two, or three buildings (two on average). These traditional houses were often situated immediately inside roadside fences, approximately $50 \mathrm{~m}$ from the Jefoure.

The households have fencing mechanisms to separate the Jefoure road and their parcel of land in a straight line (Fig. 4). The fences can be soil bunds, short, dried shrubs fastened with Enset midribs or tree outer parks, structured dry wood more than $2 \mathrm{~m}$ tall (locally called quash), and woody trees such as Eucalyptus spp. and Cupressus lusitanica (see Supplementary material 4). The fences are constructed based on agroecology, residents' experience, and resource availability. In specific areas, some residents combine fencing systems, such as soil bunds, trees, and structured wood. A soil bund may be at the inner part and will be considered an old-fashioned form of protection. Conversely, trees, such as eucalyptus, are planted outside the soil bund for raw materials. Structured wood is used in the outer fencing for beautification.

Scattered old-growth and indigenous trees can often be found on Jefoure roads (Fig. 4). These trees generously contribute to the beautification of the roads and the landscape. In the case study sites, we recorded 21 tree species. The diameter at breast height of all the recorded trees ranged from 47 to $195 \mathrm{~cm}$. These trees were large and, most notably, found in the lower and middle catchment areas of the landscape. In most localities, Afrocarpus falcatus, Croton macrostachyus, Millettia ferruginea, Cordia africana, Juniperus procera, and Olea europaea woody trees prevailed on Jefoure roads and were naturally occurring. The average number of trees per Jefoure road (without considering the road length and width) is 21 , and there are 1.5 trees per hectare. On Jefoure roads such as Cheret, 95 woody trees were recorded. The number of trees did not necessarily depend on the Jefoure road's length and width, and some roads had a larger number of trees than others (see Supplementary material 4). We rarely found trees on Jefoure roads in the upper catchment area. Although agroecology has an impact on tree growth, according to the study informants, trees such as Hagenia abyssinica found in the past. The community is not interested in preserving small trees on Jefoure roads because they have no significant socio- 

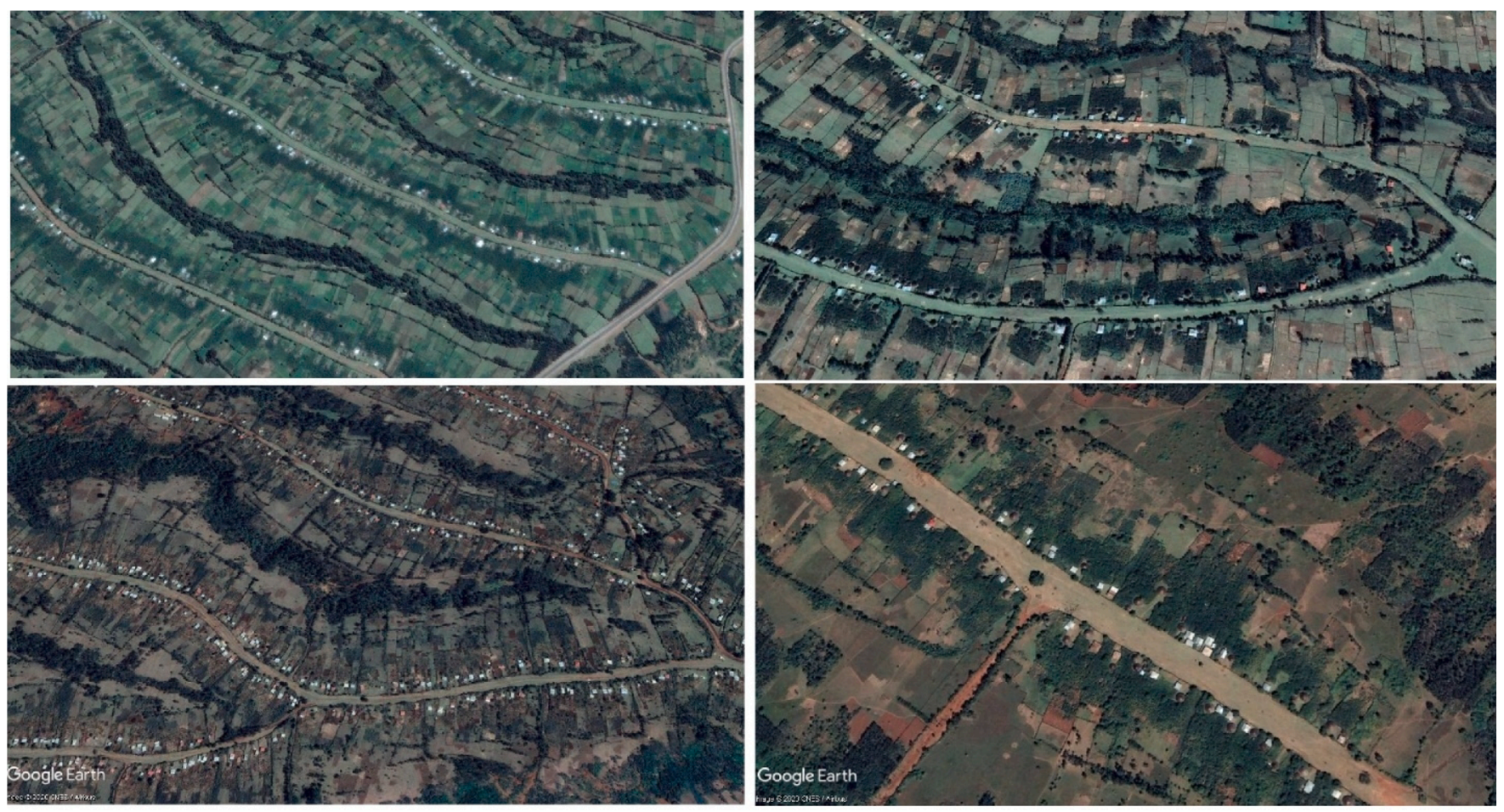

Fig. 3. The Gurage socio-ecological production landscape settlement pattern following Jefoure roads at different localities (source: Google Earth, 2019).

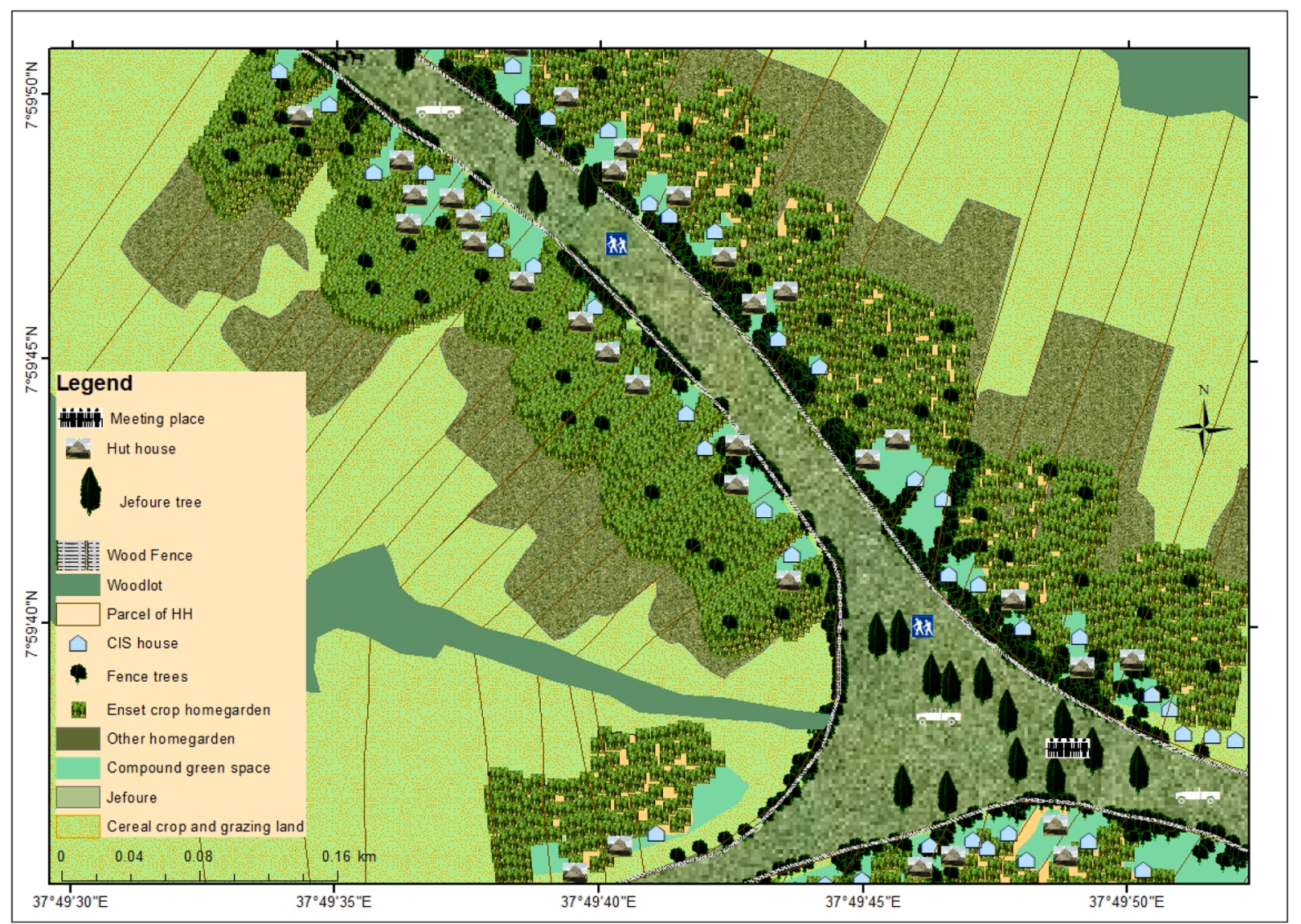

Fig. 4. Section of the Geharad Jefoure in the Enemoherna Ener district of the Gurage landscape (sketched based on orthophoto images). 
cultural value.

Based on our observations, Enset-based home garden agroforestry systems were frequently perennial crops, and fruits, spices, and vegetables were grown in backyards. The dominant perennial crops included Enset, avocado, coffee, and khat (Chatta edulis). These systems of cultivation contributed significantly to Jefoure roads becoming more scenic (Fig. 4). Enset appears green year-round and contributes to the scenic beauty of Jefoure roads, and the shaping of the landscape's settlement pattern. Next to the Enset home garden, a cereal crop-based farming system had been implemented, where food crops such as barley, teff (Eragrostis tef), wheat, and maize were grown. These cropping plots were used as grazing land for household livestock during fallow periods. In most areas, there was a stream channel at the end of the cereal crop farm and along these canals, farmers preserved natural trees and shrubs as a wood plot or planted trees and shrubs such as bamboo and eucalyptus. Large households followed this land-use pattern, and symmetric settlements were observed on opposing sides of Jefoure roads.

\subsection{Historical trends and designs of Jefoure roads}

The exact period when Jefoure roads were first designed is unknown. However, elders in the community estimated this to have been more than 400 years ago. According to the informants, their ancestors noted that the population had been very small in the past and that Jefoure roads had not been significantly developed throughout the Gurage landscape. In the past, there had been open spaces within small villages and routes that linked villages. When the population increased, settlement expansion occurred along the routes. This led to houses being built along the left and right sides of streets. Over time-symmetric land-use management, and a typical village settlement style has been formulated in every village, with minor routes subsequently joining the major streets (Fig. 4). The fencing and compound management system inbetween houses and Jefoure was introduced over time. Some households received parcels of land during land reform in the 1970s in both old and new settlement areas, and land distributors followed the same pattern in large localities.

Jefoure road widths were determined by locally assigned elders known as Yezhier Dane ("land judges"). The land judges designed settlement planning by starting from the Jefoure roads. They chose relatively upland areas for ensuring good drainage that led onto flat and relatively straight lands. The elders used an approximately $3.5 \mathrm{~m}$-long stick for measurements and assigned open routes using a minimum of seven sticks. This type of measurement and settlement had still been used until fairly recently. For example, during the land reforms in the 1970 s, land reformers used this approach in most Gurage socioecological production landscape localities. The household's parcel of land would be allocated at either the right or left side of roads.

\subsection{The spatial characteristics of Jefoure roads}

Even though the community assigned a specific width to Jefoure roads, the data extracted from the orthophoto images indicate that they were not uniform or consistent. Most of the roads stop in areas where river gorges or rigged features prevail before restarting in the next village. The maximum length of a Jefoure road that is not affected by any topographic features is approximately $20 \mathrm{~km}$, in the Enemoherna Ener district (Fig. 5). Of the total roads included in this study, 25 were longer than $10 \mathrm{~km}$, and they were in the Gumer, Geta, Ezha, and Enemoherna Ener districts. Approximately, 180 (6.2\%) roads were 5-10 km long, 296 (10\%) were 3-5 km long, and 985 (33.3\%) were 1-3 km long. Most of the Jefoure roads were shorter than $1 \mathrm{~km}$, and these constituted approximately $50 \%$ of the total road area. In total, Jefoure roads longer

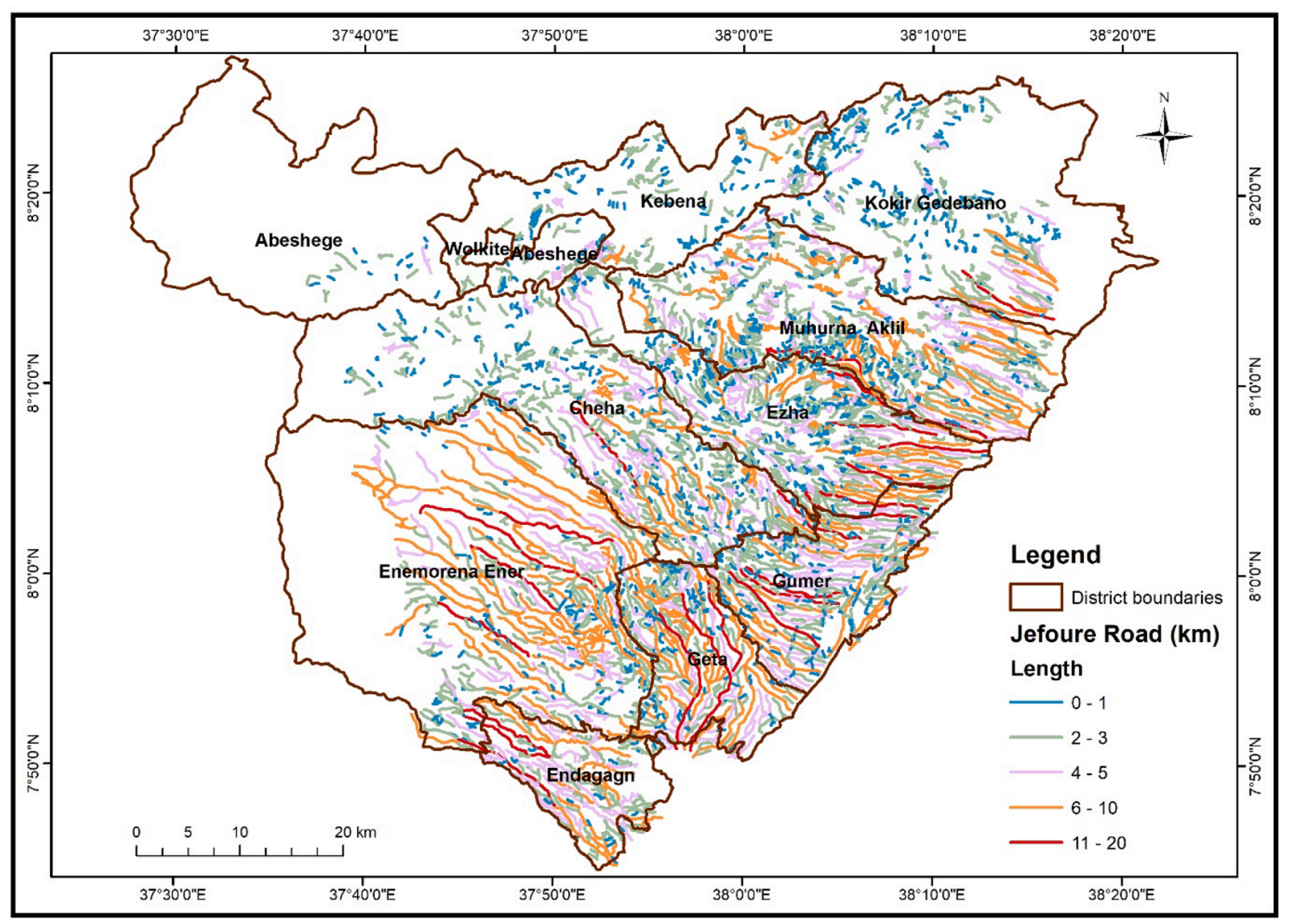

Fig. 5. Spatial distribution of Jefoure roads in Western Gurage. 
than $0.5 \mathrm{~km}$ had a combined length of $1771 \mathrm{~km}$. When we considered Jefoure roads longer than $5 \mathrm{~km}$, many were found to exist in the landscape's upper catchment parts, except for those in the Enemoherna Ener district. In the case studies, the length of roads ranged between 1.2 (Luqe) and $13 \mathrm{~km}$ (Atazo) (see Supplemental material 4). The average length of the selected Jefoure roads was approximately $6 \mathrm{~km}$. The road length correlated with elevation differences and slope gradients. Longer roads included more considerable elevation differences and higher slope gradients.

Data extracted from the orthophotos indicated that the existing Jefoure roads have diverse dimensions. However, most are extensive than $5 \mathrm{~m}$ and reached up to $87 \mathrm{~m}$. Although wide breadth roads are generally short, the most expansive $(87 \mathrm{~m})$ among them is Yadazer Jefoure road, in the Mihurna Aklil district. Locations that resembled a square in Jefoure roads have more than this extent and can range up to a radius of $150 \mathrm{~m}$. The average Jefoure road in the study area is $23 \pm 8 \mathrm{~m}$. Of the total roads included, approximately $12.4 \%$ are $5-10 \mathrm{~m}$ wide. Roads ranging between 30 and $40 \mathrm{~m}$ represented approximately $32.1 \%$. Many Jefoure roads are 20-30 m wide, covering approximately $41.4 \%$ of the total area. Approximately, $3.2 \%$ of roads are $40-50 \mathrm{~m}$ wide. Roads wider than $50 \mathrm{~m}$ are rare (an estimated $0.1 \%$ of the total road). The spatial data showed that many Jefoure roads with a width $>30 \mathrm{~m}$ is located in the Enemoherna Ener, Ezha, Gumer, and Cheha districts. In the case studies, Jefoure roads wider than $60 \mathrm{~m}$ are found in the Yadazer, Inagera, Luqe, Dength, Demberi, and Geharard areas (see Supplemental material 4).

Jefoure roads have hierarchies based on functionality, width, and length. A local categorization mechanism is in place for observing this. Wide and long roads are referred to locally as Wur Ema, meaning the "main road". This type of road connects districts and bypass several villages (Fig. 6). Jefoure roads wider than $30 \mathrm{~m}$ and longer than $10 \mathrm{~km}$ are for the most part categorized as Wur Ema. Some roads that are recognized by community elders as being associated with historical events, such as Geharad in Enemoherna Ener district, Aegera and Bulecho in the Gumer district, Ayanete in the Cheha district, Fereze in the Geta district, Desene in the Ezha district, and Yadazer in the Mihurna Aklil district, can be categorized as this type. These roads, which are relatively shorter in length and have a narrower breadth than the Wur Ema, are known simply as Ema ("road"). This type of road often connects villages. Roads that connect minor Jefoure roads with widths between 20 and $30 \mathrm{~m}$ and lengths between 5 and $10 \mathrm{~km}$ are categorized as Ema. There are also minor roads that connect two parallel Jefoure roads, which can be labeled as local roads. These different types of routes provide access to resources, such as water and grazing lands.

\subsection{Physical geographical characteristics of the Jefoure roads}

Despite the difference in the lengths and widths of Jefoure roads, they were found in all elevation ranges of the Gurage socio-ecological production landscape. The spatial features extracted from the orthophotos showed that the lower elevation areas had short and wide Jefoures. Approximately $24.7 \%$ of roads were found between 1501 and $2000 \mathrm{~m}$ above sea level; this elevation range covered $37.7 \%$ of the landscape (Fig. 7). The elevation ranges from 2001 to $2500 \mathrm{~m}$ covered $25.6 \%$ of the landscape, and approximately $33.5 \%$ of Jefoure roads were found within this range. The elevation gradients between 2501 and $3000 \mathrm{~m}$ covered $21.2 \%$ of the landscape, and nearly $31.5 \%$ of roads were extracted within this range. The high mountainous part of the landscape, where elevation is $>3000 \mathrm{~m}$, covered only $7.2 \%$ of the landscape and included roughly $10.3 \%$ of Jefoure roads. The elevation differences among the 21 roads examined in this study were diverse (see Supplemental material 4). These differences ranged from the lowest in Lencha $(13 \mathrm{~m})$ to the highest in Kentuat (403 m). Most of the lower elevation Jefoure roads exhibited smaller elevation differences, except for selected

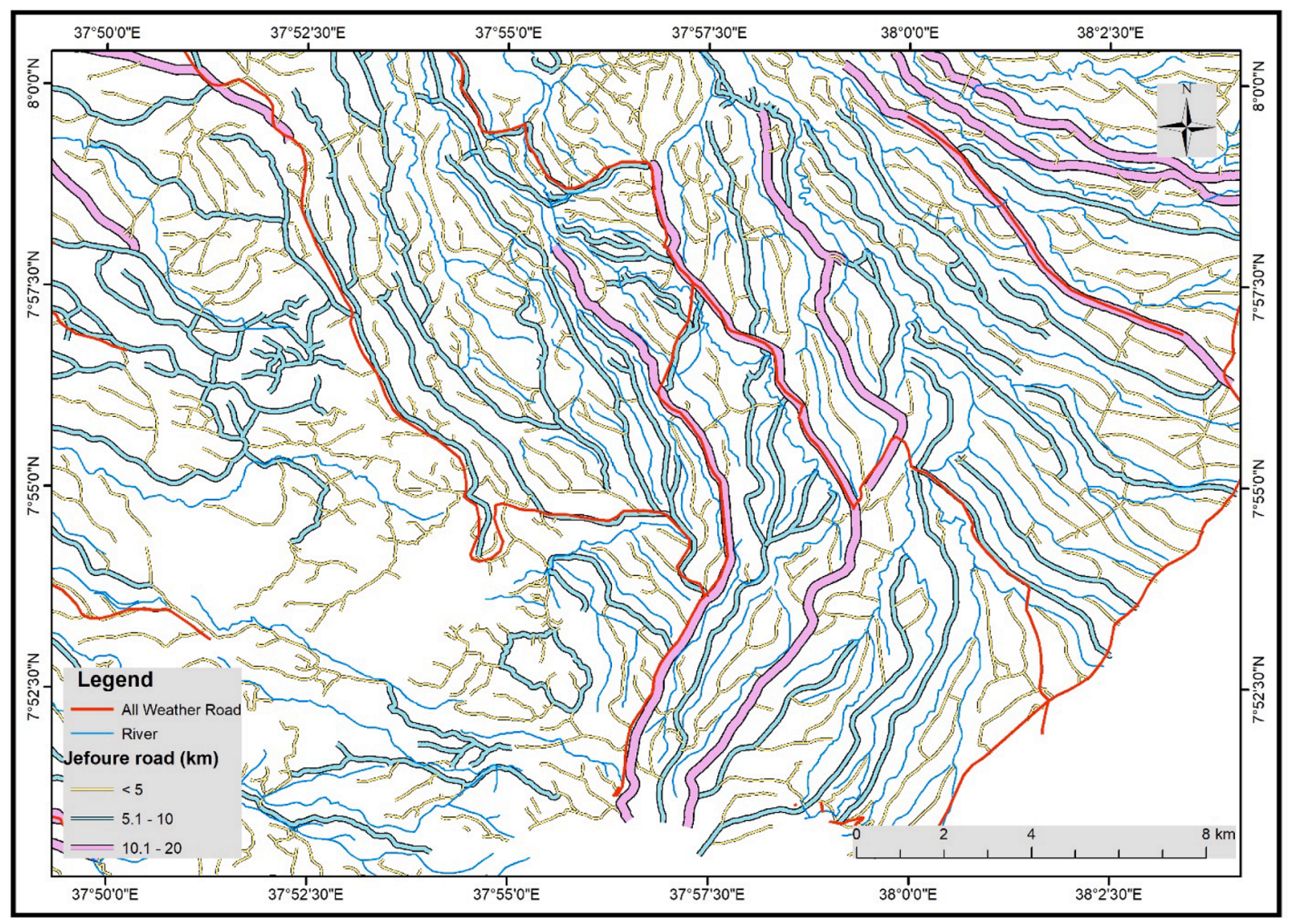

Fig. 6. The Jefoure cultural road hierarchy in the Geta district of Western Gurage. 


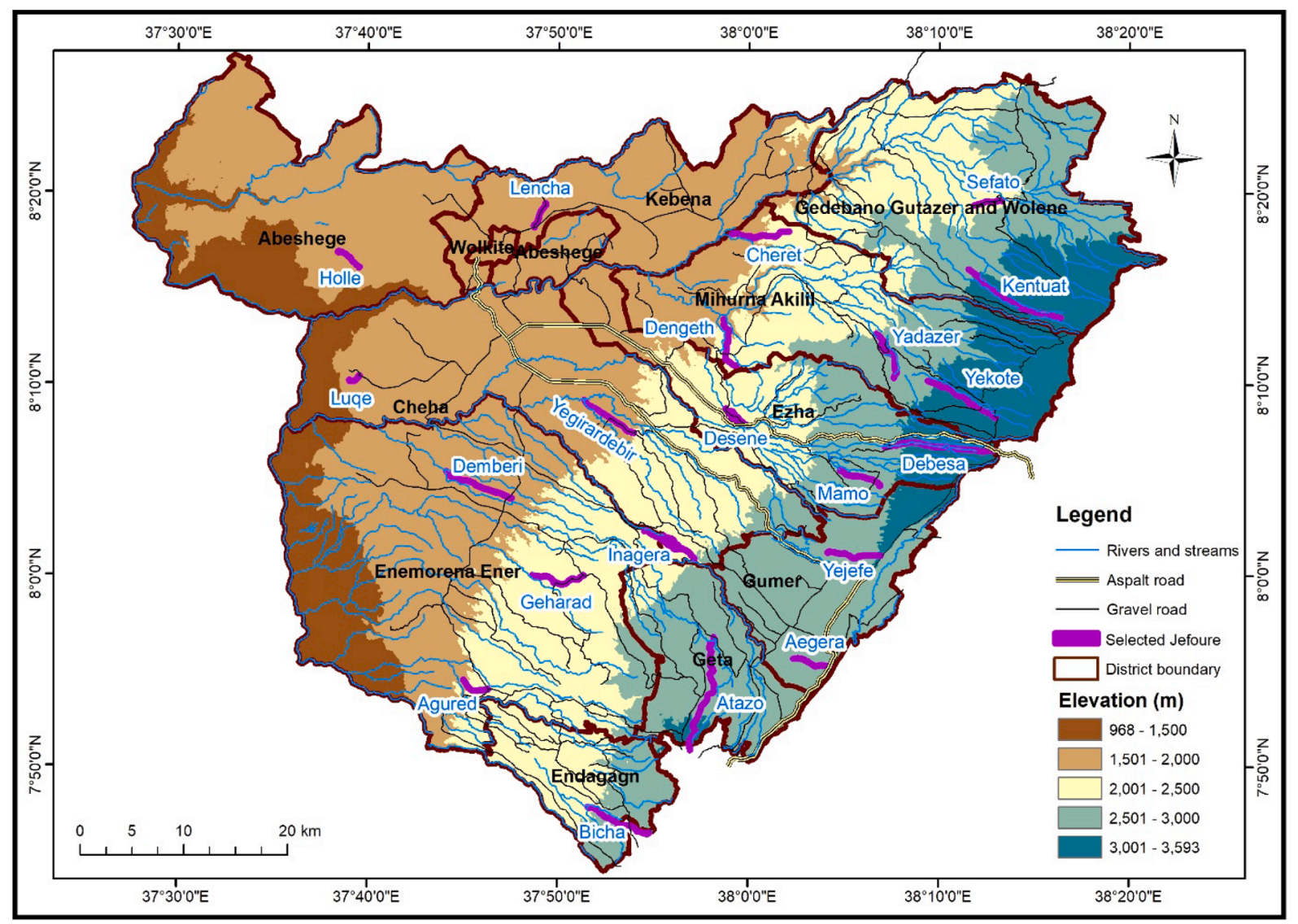

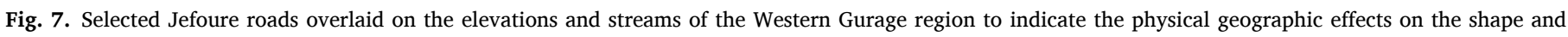
orientation of roads.

roads such as Cheret and Yegirardebir. Notably, Jefoure roads found above $2000 \mathrm{~m}$ indicated large elevation differences.

A slope difference of $0^{\circ}-64^{\circ}$ was observed in the Western Gurage socio-ecological production landscape. Although the local land judges allocated Jefoure roads in relatively upland landscapes and flat areas, the extracted data showed that roads had slopes ranging between $0 \%$ and $62 \%$ (see Supplemental material 3). Approximately $21.5 \%$ of Jefoure roads were located in flat areas ( $0 \%-5 \%)$, and roughly $32.2 \%$ had slopes ranging between $5 \%$ and $10 \%$. A large portion of roads (33.8\%) had slope rises of $10 \%-20 \%$, and approximately $12.4 \%$ had slope ranges of $20 \%-40 \%$. The remaining $1.1 \%$ of roads had slopes $>40 \%$. For selected Jefoure roads, the slope differences ranged between $6 \%$ and $47.4 \%$ (see Supplementary material 4).

There were diverse orientations among Jefoure roads, and most had east-to-west direction orientations (Fig. 7). Eight of the 21 case study sites had an approximate east-to-west orientation (see Supplementary material 4). Six Jefoure roads stretched southeast and were shaped in an approximately northwest direction. These roads followed the drainage patterns of the landscape, similar to other physical factors.

Jefoure roads have a diverse range of shapes. Most are linear and straight and are present in the landscape's upper catchment areas (Fig. 7). Others have curvilinear and irregular shapes. Most of the middle-altitude roads found in the Mihurna Aklil, Ezha, and Gedebano Gutazer Wolene districts have irregular shapes influenced by the landscape's topographic features. Of the 21 case study sites, 10 roads had approximately straight-line shapes (see Supplemental material 4). Not all were precisely straight as shown in Fig. 7; nine of the selected roads had both straight and curvilinear-shaped sections.

In the case study, some of the Jefoure roads' starting points were main roads (Fig. 7). Three of these began with another Jefoure road as a minor street and then increased in width. In some cases, communal land served as the beginning of a Jefoure road, e.g., the Yadazer, Sefato, Inagera, and Bercha roads (see Supplemental material 4). According to the case study, wetlands served as the starting points for Jefoure roads, with communities settling in the immediate upland areas. The Demberi and Yekote Jefoure roads' current starting points began in conjunction with cereal cropland use, which had initially been landscapes used for livestock grazing. Afroalpine vegetation was the early land cover shared by the most notable Jefoure roads in the upper catchment areas of Mihur Aklil and Gedebano Gutazer Wolene districts. Five of the roads in the case study ended with stream canals (see Supplementary material 4). The Yadazer, Inagera, and Kentuat Jefoure roads stop when they reach gullies created by soil erosion. Other roads end when they reach a communal forest.

\subsection{Socio-cultural and ecological characteristics of Jefoure roads}

According to the informants, the community's primary motivation when allocating part of the landscape to a Jefoure road is to enable free physical access for people and livestock. Jefoure roads' primary objective is to serve as natural transport modes, which they have been able to do for an extended period with continuous management. When motorized vehicles were introduced to the landscape, roads enabled vehicle accessibility, except in localities, they need drainages and are found in high slopes. However, the heavy trucks that transport timber products are degrading Jefoure roads in some localities, particularly during the rainy seasons.

Over time, Jefoure roads have enabled communities to practice social and cultural activities, such as spiritual and religious practices (e.g., making bonfires during Meskel festivals), local judicial services (Fig. 8), and marriage and mourning ceremonies, while also serving as playgrounds and grain threshing floors (see Suplementary material 5). 


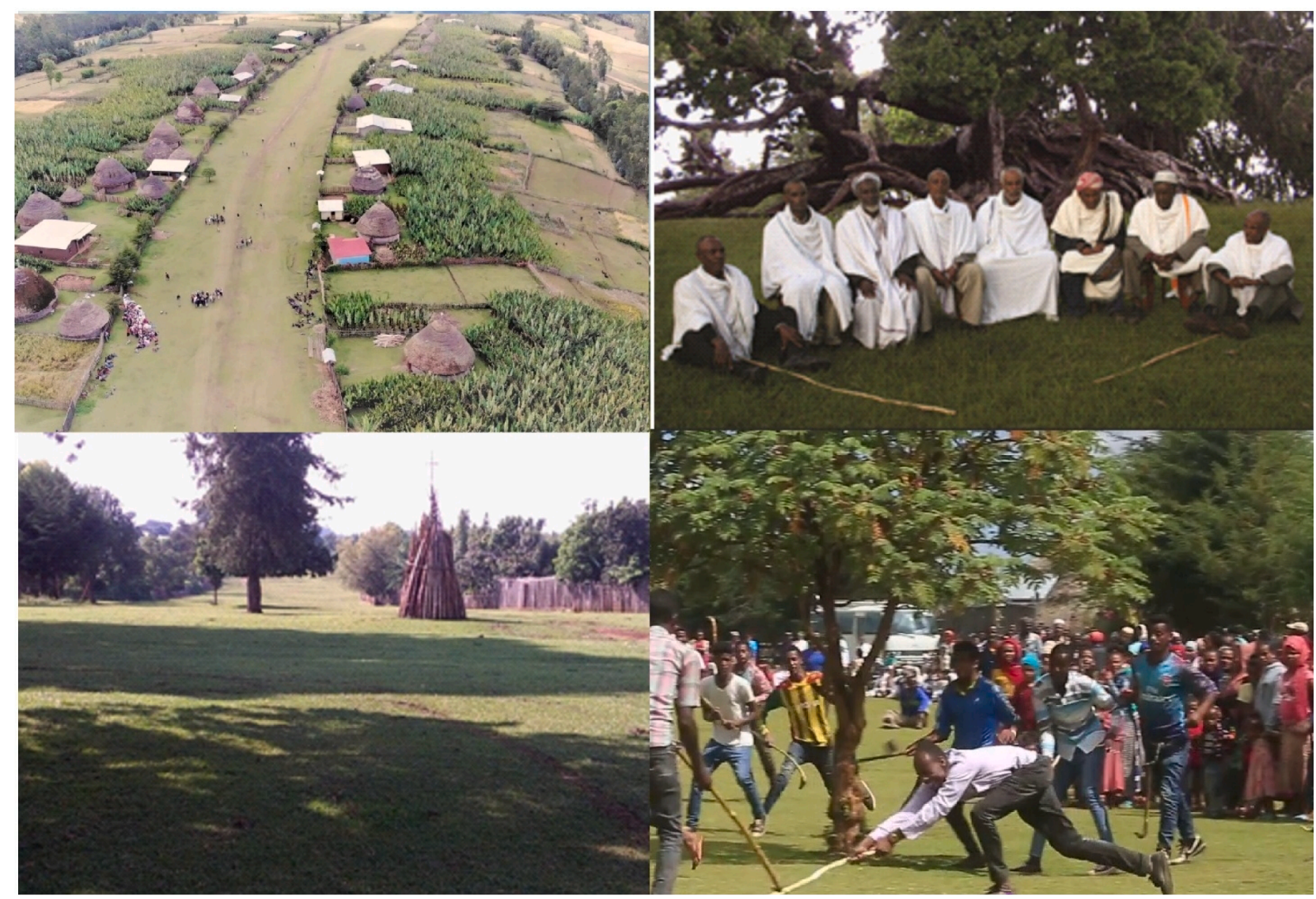

Fig. 8. Socio-cultural practices that take place on Jefoure roads (source: Tourism Culture and Sport Office (Gurage zone)).

Currently, these roads enhance the socio-ecological production landscape's scenic beauty and distinguish the landscape from other cultural settings in Ethiopia. The roads support the provisioning of services, such as livestock feed production from the roads and biomass from along the fences for firewood and timber. These grass-covered roads, which host vascular trees and side fences, can also regulate local temperature, climate, water flow, and soil erosion. Since there is no reserved area for recreation, Jefoure roads also serve as a space in which to spend free time and are used during annual festivals.

\subsection{Community-based management of Jefoure roads}

Preserving Jefoure open spaces is one of the main issues observed by the general and local councils of community elders. The elders in each village control the overall management of these roads. When villagers add parts of a road to their parcel of land illegally, the community will inform the local council. A type of conflict resolution called Gurda ("covenant") is implemented, where the council will review issues and subsequently implement sanctions if required. Penalties can take the form of being prohibited from participating in social services or having to pay a fixed amount in either money or items. As such, community members do not often make use of Jefoure roads beyond the extent of their farmland and are disinclined to commit illegal acts on these roads. In some localities, no-one may even plant a tree on their immediate Jefoure road prior to receiving approval from the community.

Jefoure roads are under continuous management in some localities. There is competition among villagers in terms of the quality of Jefoure management. According to informants, Jefoure roads that are wide, long in length, have lower slope ranges, run in a straight direction, have scattered trees and sound fencing systems are considered good quality Jefoures. The roads that fulfill these criteria are considering exemplary, and the communities situated alongside these roads will attempt to manage their villages accordingly. This experience-sharing between villagers has resulted in the development of a common type of Jefoure road within the landscape. In contrast, some of the longest roads have recently been shortened or have had their width narrowed by road construction, initiated by both government and community organizations. Gravel roads are being constructed by excavating the grass within many areas of the landscape, which could have a significant impact on the sustainability of Jefoure roads.

\section{Discussion}

\subsection{Complementary approaches to landscape characterization}

Mapping landscape spaces using manual and modern technology enables landscape architects to describe, understand, and interpret landscapes' spatial/visual properties (Liu \& Nijhuis, 2020). This mapping can support strengthening the body of knowledge about spatial characteristics in landscape architecture. Since the landscape is a result of the action and interaction of natural and/or human factors (Roe, 2007), roads and their surroundings can be considered as a landscape (Kołodziej, 2017). In this study, we considered Jefoure roads as part of the Gurage cultural landscape and characterized them based on landscape principles. According to Groom (2005), in a comprehensive analysis of landscape typologies in Europe, six dimensions can be addressed in landscape-type mapping and landscape character assessment. These are the biophysical dimensions, landscape ecological issues, socio-economic technical dimensions, historical dimensions, humanaesthetic dimensions, and user participation and policy dimensions. Therefore, we considered the historical, spatial, physical geographical, socio-cultural, ecological, and management dimensions of landscape characterization for a better understanding of Jefoure roads in the Gurage socio-ecological production landscape in Ethiopia. 
Landscape research's multidisciplinary nature means that many different systems and methods for landscape identification and classification are in place (Simensen et al., 2018; Tieskens et al., 2017). There is no single approach with which to address all landscape typology dimensions, however, and significant trade-offs and substantial differences exist between landscape characterization methods (Groom, 2005). Combining complementary methods that are explicitly directed at user needs may compensate for limitations and trade-offs within single methods (Simensen et al., 2018). As we considered the different dimensions of the Jefoure road landscape, our methodological approaches also differed. We used "holistic" character assessment approaches to characterize the socio-cultural aspects of the landscape. The ecological and land-use-related properties of the landscape employed for characterization related to land-use management and spatial extent. The physical landscape characterization approaches depict the road landscape on the region's natural elements, e.g., topographic and hydrological structures. These characterization approaches support exploring the road landscape from diverse perspectives, such as land-use management, road transport, socio-cultural aspects, physical geography, landscape ecology, and landscape planning.

\subsection{Co-evolution of societies and the development of a multifunctional landscape}

People have long since used the natural world for hunting, harvesting, and to develop grazing systems for domestic animals using arable land. Over time, people began approaching nature more functionally and rationally (Vos \& Meekes, 1999). As a result, many cultural landscapes have been developed through the continuous restructuring of land; people became accustomed to using land and structuring it better spatially, based on a variety of societal demands (Antrop, 2005). As growing mobility increased worldwide, roads, whether traditional or semi-technology-based, were developed into the 18th and 19th centuries (Berechman, 2003). The social, cultural, and political significance of roads and their surroundings served as drivers and symbols of human development (Grazuleviciute-Vileniske \& Matijosaitiene, 2010). As the result of social, economic, and technological changes, traditional landuse practices have intensely altered and homogenizing of landscape (e.g., Mono cropping and urbanization) has been started (Kizos \& Vlahos, 2012; O'farrell \& Anderson, 2010).

Multifunctionality has gained increasing interest in areas of science and policy (Renting et al., 2009; European Commission, 2015). Multifunctional concepts in the context of land development emerged because of a need to satisfy a range of demands on particular landscapes (Wiggering, Müller, Werner, \& Helming, 2003). Ecological landscape planning such as greenways, ecological networks, and ecological infrastructures can suitably address the demands of multifunctional landscapes (Kato \& Ahern, 2009; Phillips, Bullock, Osborne, \& Gaston, 2020). Multiple uses also facilitate the efficient use of time and space, which is a particularly attractive feature for urban landscapes, where space is limited and a land mosaic is fine and heterogeneous (Kato \& Ahern, 2009). The Jefoure road landscape is a multifunctional landscape that evolved based on the indigenous knowledge of native Ethiopians. The roads are designed in a manner that follows the landscape's natural surroundings. This approach allows for the sustainable development of the landscape with limited adverse ecological effects. Jefoure roads are multifunctional because of their preserved grass cover, old-growth trees, fence management, and trees planted alongside them, which have been well-integrated with the surrounding environment and are tended to by continuous community-based management.

Recently, changes have been perceived as threatening in terms of causing the loss of diversity, coherence, and identity linked to traditional cultural landscapes (Antrop, 2005). There is a need to recognize the importance of traditional landscapes, and to mainstream policy and decision-making regarding sustainable management before these landscapes disappear completely (UNU-IAS \& IGES, 2016).

\subsection{Improve policy and its implementation to conserve cultural landscapes more efficiently}

Many countries preserve rich natural and cultural heritage values as part of people's daily lives and provide authoritative and socially cohesive power to live. However, these heritages are under threat, mainly due to changing socio-cultural values, globalization, and a lack of legal bounds (Mercy, Cyril, \& Brendan, 2011). With the pace of development, vast numbers of cultural landscapes and archaeological sites are lost each year (Grant, Gorin, \& Fleming, 2002). Road landscapes can be valuable not only because they serve as interconnections with one particular event, but because they also reflect entire periods of development linked to particular social groups (Grazuleviciute-Vileniske \& Matijosaitiene, 2010). Similarly, Jefoure roads serve as indicators of the long-term history, efforts, and knowledge of communities living within the landscape. Road construction associated with socioeconomic changes is the primary challenge faced by Jefoure roads and has an impact on their direction, shape, hierarchies, and beginning and ending points. Attempts to reconcile aesthetics, visual issues, human needs, and ecological aspects related to roads are encouraged in the consideration of these landscapes.

Recently, Ethiopia implemented the National Cultural Policy (ratified in 2015), Research and Conservation of Cultural Heritage Proclamation (209/2000), and the Environmental Impact Assessment Proclamation (299/2002) to ensure the protection and management of its cultural heritage. Despite the different laws in place regarding the management and protection of cultural heritage, most are not fully implemented. Policies and procedures require all regional and federal culture and tourism offices to take cultural management concerns into account in the programs and projects they are responsible for. The system involves cooperation among federal, state, and local governments, and between the public and private sectors. The country's cultural values, including cultural landscapes, are not entirely nominated and registered and, at best, tend to focus on tangible historical heritage sites. This results in the bulk of potential heritage sites lacking legal grounds for receiving priority protection within national development strategies. Therefore, the Authority of Research and Conservation of Cultural Heritage in Ethiopia needs to work with regional and local tourism and culture offices to fast-track legislative backing to stop the deterioration of cultural assets, including Jefoure landscapes. Effective implementation and enforcement of existing policies and regulations are needed not only in cultural sectors but also in the context of other stakeholders. Thus, cooperation, partnership, and the increased involvement of all stakeholders, including government and non-government organizations and private sectors, should be mandatory to protect and manage existing cultural landscapes.

\subsection{International recognition of the Jefoure landscape}

Roads undoubtedly influence environments and are typically accepted as part of a landscape. Cultural roads represent traditional human settlements and land-use, and, as such, cultural/human interaction with the environment (Grazuleviciute-Vileniske \& Matijosaitiene, 2010). According to Mauch and Zeller (2008), various roads and their environments, whether designed for roadside aesthetics or as fast and efficient transportation, are the outcomes of historical negotiations. Such roads can contribute to the integrity, permanency, and expressivity of treasured cultural landscapes. These roads reflect specific periods and ideas linked to road development and the socio-cultural and political conditions that provoked them and, accordingly, can have historical implications (Grazuleviciute-Vileniske \& Matijosaitiene, 2010). Road landscapes can be regarded as a type of cultural heritage, demonstrating positive quality interactions between people and their surrounding environment. Not only the aesthetic and harmonious aspects of road landscapes are important in terms of cultural heritage; the roads themselves can become a form of cultural heritage because they represent 
technical and historical phases of development (Mauch \& Zeller, 2008).

The Jefoure road landscape originated based on social, economic, and administrative norms, which evolved into the present based on interactions between people and nature. This road landscape can be categorized as an organically evolved landscape of the UNESCO cultural landscapes (UNESCO, 2008). The Jefoure road landscape qualifies at least three main criteria as mixed natural and cultural heritage, i.e., criterion (iii), to bear a unique or at least exceptional testimony to a cultural tradition which is living; criterion (v), to be an outstanding example of human settlement and land-use which is representative of a culture, or human interaction with the environment; and the criterion (vi), to be directly associated with living traditions or with beliefs. The Jefoure cultural roads were created with deliberate intention and organically formed over the course of time and have social, cultural, spiritual, aesthetic, and ecological significance. They must be considered as cultural heritage, together with the surrounding environments and other cultural assets of the community to enhance their conservation and sustainable management.

To operationalize the heritagization of the landscape, a legalized committee that includes various stakeholders must be established to further identify and map the main features of the cultural landscape for nomination. Boundary delineation, a heritage site management plan, legal ownership transferal documents, and documentation (text, images, maps, and video) must be prepared and submitted to the World Heritage Center. This study provides baseline information regarding the registration process.

\section{Conclusion}

Various landscape dimension characteristics will enable a better understanding of landscapes from different perspectives and can help to establish sustainability solutions. Cultural landscapes are not created at an exact or specific time but through generational interaction with nature. Accordingly, it can be difficult to understand any single cultural object using a specific dimension of landscape characteristics since comprehensive documentation may not be available. In this study, we focused on Jefoure cultural roads in the Gurage socio-ecological production landscape of Ethiopia. We characterized cultural roads according to dimensions such as general landscape characteristics, spatial and physical geographical dimensions, design principles and layout, sociocultural values, and management aspects. These approaches allowed us to understand the landscape from different perspectives.

Cultural roads influence the landscape's settlement patterns and continue to play social and cultural roles in present-day society. The Gurage socio-ecological production landscape and its Jefoure cultural roads is a continually evolving landscape that is closely associated with traditional ways of life. The local community designed Jefoure roads without prior knowledge of landscape architecture. These cultural roads play a significant role in terms of connecting rural people to cities, in fulfilling various socio-cultural and ecological roles, enhancing settlement patterns, and facilitating infrastructure provisions. Design principles and elements that could be taken lesson form Jefoure roads include preserving grass cover (where traffic is limited), road verge development, preserving old-growth trees, allocating spaces between houses and the nearest by roads, designing roads that follow the physical geographical landscape, and community participation in the management of roads.

Cultural landscapes originated within the contexts of social, economic, administrative, and religious norms and have evolved into their present status relative to the natural environment. Roads and their elements can become cultural heritage objects themselves because they represent technical or historical development phases. These roads and their evolving cultural landscapes require local and international recognition as heritage sites to ensure their sustainability. This study includes valuable empirical information about road features and can support the local community, land-use planners, decision-makers, and conservation initiatives in addressing current challenges, and enhance the sustainability of Jefoure landscapes. It may also inform and inspire other studies focusing on similar cultural roads that are on the verge of disappearing.

\section{CRediT authorship contribution statement}

Conceptualization, data collection, formal analysis, methodology development, visualization and draft writing were under taken by the first author. The second author finds scholarship fund, supervised the overall works and review and edit the manuscript.

\section{Declaration of Competing Interest}

The authors declare that they have no known competing financial interests or personal relationships that could have appeared to influence the work reported in this paper.

\section{Acknowledgments}

The key interviews and FDGs in this study were conducted with the kind cooperation of the Gurage Zone office of Tourism Culture Sport and Wolkite University in Ethiopia. The financial support was obtained from the Overseas Researcher Postdoctoral Fellowship of the Japan Society for the Promotion of Science. The United Nations University Institute for the Advanced Study of Sustainability and the Institute of Global Environmental Strategies in Japan provided logistic support. Therefore, we gratefully acknowledge all these organizations and the financial support rendered to the first author.

\section{Appendix A. Supplementary data}

Supplementary data to this article can be found online at https://doi. org/10.1016/j.landurbplan.2021.104078.

\section{References}

African World Heritage. (2018). Traditional Cultural Landscapes [Accessed 23 October 2020] https://www.africanworldheritagesites.org/cultural-places/traditional-cultur al-landscapes.html.

Antrop, M. (2005). Why landscapes of the past are important for the future. Landscape Urban Planning, 70(1-2), 21-34. https://doi.org/10.1016/j. landurbplan.2003.10.002.

Berechman, J. (2003). Transportation - Economic aspects of Roman highway development: The case of Via Appia. Transportation Research Part A: Policy and Practice, 37(5), 453-478. https://doi.org/10.1016/S0965-8564(02)00056-3.

Borrell, J. S., Biswas, M. K., Goodwin, M., Blomme, G., Schwarzacher, T., HeslopHarrison, J., et al. (2019). Enset in Ethiopia: A poorly characterized but resilient starch staple. Annals of Botany, 123(5), 747-766. https://doi.org/10.1093/aob/ mcy214.

Brandt, S. A., Spring, A., Hiebsch, C., McCabe, J. T., Tabogie, E., Diro, M., et al. (1997), The "tree against hunger" Enset-based agricultural systems in Ethiopia. American Association for the Advancement of Science with Awassa Agricultural Research Center. Kyoto University Center for African Area Studies and University of Florida, American Association for the Advancement of Science.

Brenna, B., Larsen, J. K., \& Hvattum, M. (2011). Routes, Roads and Landscapes (p. 272). Routledge.

Campolo, D., Bombino, G., \& Meduri, T. (2016). Cultural landscape and cultural routes: Infrastructure role and indigenous knowledge for a sustainable development of Inland Areas. Procedia - Social and Behavioral Sciences, 223(10), 576-582.

Central statistical Agency (CSA). (2009). Population and house census report of Ethiopia. Ethiopia: Addis Ababa.

Ethiopian Mapping Agency (EMA). (2017). Aerial photography quality control. SLMP south block 1 and 2 project. Ethiopia: Addis Ababa.

European Commission. (2015). Towards an EU research and innovation policy agenda for nature-based solutions and re-naturing cities. Brussels, Belgium: European Commission.

Fisher, B., Turner, R. K., \& Morling, P. (2009). Defining and classifying ecosystem services for decision making. Ecological Economics, 68, 643-653. https://doi.org/ 10.1016/j.ecolecon.2008.09.014.

Grant, J., Gorin, S., \& Fleming, N. (2002). In The archaeology coursebook. An introduction to study skills, topics, and methods (pp. 196-203). London and New York: Routledge, Taylor and Francis Group.

Grazuleviciute-Vileniske, I., \& Matijosaitiene, I. (2010). Cultural heritage of roads and road landscapes: Classification and insights on valuation. Landscape Research, 35, 391-413. https://doi.org/10.1080/01426397.2010.486856. 
Groom, G. (2005). Methodological review of existing classifications. In D. M. Wascher (Ed.), European landscape character areas - Typology, cartography and indicators for the assessment of sustainable landscapes. Final ELCAI project report, landscape Europe (pp. $32-45)$.

Gurage zone finance and economic development (GZFED). (2018). Gurage zone 2017/18 socio-economic abstract. Ethiopia: Wolkite.

Hartel, T., Fischer, J., Campeanu, C., Milcu, A. I., Hanspach, J., \& Fazey, I. (2014). The importance of ecosystem services for rural inhabitants in a changing cultural landscape in Romania. Ecology and Society, 19(2), 42. https://doi.org/10.5751/ES06333-190242.

Hazeu, G. W., Metzger, M. J., Mücher, C. A., Perez-Soba, M., Renetzeder, C., \& Andersen, E. (2011). European environmental stratifications and typologies: An overview. Agriculture Ecosystems and Environment, 142(1-2), 29-39. https://doi.org/ 10.1016/j.agee.2010.01.009.

Herzog, F. (1998). Streuobst: A traditional agroforestry system as a model for agroforestry development in temperate Europe. Agroforestry Systems, 42, 61-80. https://link.springer.com/article/10.1023/A:1006152127824.

Hudson, G. (1996). Essays on Gurage language and culture: Dedicated to wolf Leslau on the occasion of his 90th birthday (p. 239). Wiesbaden, Germany: Otto Harrassowitz.

Huntsinger, L., \& Oviedo, J. L. (2014). Ecosystem services are social-ecological services in a traditional pastoral system: The case of California's Mediterranean rangelands. Ecology and Society, 19(1), 8. https://doi.org/10.5751/ES-06143-190108.

Japan Satoyama Satoumi Assessment (JSSA). (2010). Satoyama-satoumi ecosystems and human wellbeing: Socio-ecological production landscapes of Japan (summary for decision makers). Tokyo: United Nations University.

Kato, S., \& Ahern, J. (2009). Multifunctional landscapes as a basis for sustainable landscape development. Journal of The Japanese Institute of Landscape Architecture, 72 (5), 799-804. https://doi.org/10.5632/jila.72.799.

Kizos, T., \& Vlahos, G. (2012). The evolution of the agricultural landscape. In Reclaiming the Greek landscape (pp. 133-143). MED-INA, Papayiannis Thimios, Howard Peter.

Kołodziej, A. (2017). Landscape routes as an infrastructural core of cultural landscapes; their distinctive role for the character of region. IOP Conference Series Materials Science and Engineering, 245(4), Article 042053. https://doi.org/10.1088/1757899X/245/4/042053.

Liu, M., \& Nijhuis, S. (2020). Digital methods for mapping landscape spaces in landscape design. Journal of Digital Landscape Architecture, 5-2020, 634-645. https://doi.org/ $10.14627 / 537690065$

Mauch, C., \& Zeller, T. (2008). Introduction. In C. Mauch, \& T. Zeller (Eds.), The World Beyond the windshield: Roads and landscapes in the United States and Europe (pp. 1-13). Athens, OH: OhioUniversity Press.

Mercy, N. U., Cyril, E. C., \& Brendan, A. E. (2011). Globalization of cultural heritage: Issues, impacts, and inevitable challenges for Nigeria. Library Philosophy and Practice (e-journal). Paper 674

Nida, W. (2000). Fanonet: Ethnohistorical notes on the Gurage Urban migration in Ethiopia. Ufahamu: A Journal of African Studies, 28, 2-3.

O'farrell, P. J., \& Anderson, P. M. (2010). Sustainable multifunctional landscapes: A review to implementation. Current Opinion in Environmental Sustainability, 2, 59-65. https://doi.org/10.1016/j.cosust.2010.02.005.

Parrott, L., \& Meyer, W. S. (2012). Future landscapes: Managing within complexity. Frontiers in Ecology and the Environment, 10, 382-389. https://doi.org/10.1890/ 110082.

Phillips, B. B., Bullock, J. M., Osborne, J. L., \& Gaston, K. J. (2020). Ecosystem service provision by road verges. Journal of Applied Ecology, 57(3), 488-501. https://doi. org/10.1111/1365-2664.13556.

Plieninger, T., Bieling, C., Ohnesorge, B., Schaich, H., Schleyer, C., \& Wolff, F. (2013). Exploring futures of ecosystem services in cultural landscapes through participatory scenario development in the Swabian Alb, Germany. Ecology and Society, 18(3), 39. https://doi.org/10.5751/ES-05802-180339.

Plieninger, T., van der Horst, D., Schleyer, C., \& Bieling, C. (2014). Sustaining ecosystem services in cultural landscapes. Ecology and Society, 19(2), 59. https://doi.org/ 10.5751/ES-06159-190259.

Rabbinge, R., \& Bindraban, P. S. (2012). Making more food available: Promoting sustainable agricultural production. Journal of Integrative Agriculture, 11, 1-8. https://doi.org/10.1016/S1671-2927(12)60777-9.

Renting, H., Rossing, W., Groot, J., Van der Ploeg, J. D., Laurent, C., Perraud, D., et al. (2009). Exploring multifunctional agriculture. A review of conceptual approaches and prospects for an integrative transitional framework. Journal of Environment Management, 90, S112-S123. https://doi.org/10.1016/j.jenvman.2008.11.014.

Roe, M. H. (2007). The European landscape convention: A revolution in thinking about "cultural landscapes. Journal of Chinese Landscape Architecture, 23(143), 10-15.
Rotondo, F. (2016). Studying cultural territorial systems: Introduction. In F. Rotondo, F. Selicato, V. Marin, \& J. Lopez Galdeano (Eds.), Cultural territorial systems. Springer geography. Cham: Springer. https://doi.org/10.1007/978-3-319-20753-7_1.

Sahle, M., Yeshitela, K., \& Saito, O. (2018). Mapping the supply and demand of Enset crop to improve food security in Southern Ethiopia. Agronomy for Sustainable Development, 38, 7. https://doi.org/10.1007/s13593-017-0484-0.

Saito, O., Subramanian, S. M., Hashimoto, S., \& Takeuchi, K. (2020). Introduction: Socioecological production landscapes and seascapes. In O. Saito, S. Subramanian, S. Hashimoto, \& K. Takeuchi (Eds.), Managing socio-ecological production landscapes and seascapes for sustainable communities in Asia. Science for sustainable societies. Singapore: Springer. https://doi.org/10.1007/978-981-15-1133-2_1.

Schepaschenko, D., See, L., Lesiv, M., et al. (2019). Recent advances in forest observation with visual interpretation of very high-resolution imagery. Surveys In Geophysics, 40, 839-862. https://doi.org/10.1007/s10712-019-09533-z.

Shack, W. A. (1966). The Gurage: A people of the enset culture. African ethnographic studies of the 20th Century Vol. 59. Oxford University Press.

Simensen, T., Halvorsena, R., \& Erikstad, L. (2018). Methods for landscape characterisation and mapping: A systematic review. Land Use Policy, 75, 557-569. https://doi.org/10.1016/j.landusepol.2018.04.022.

Tadesse, M. (2009). Hazo: A political history of the people in Gedebano, Gutazer, Welene and Agemjay localities of the Gurage land, Ethiopia (to 1991). Ethiopia: Hawassa.

Takeuchi, K. (2010). Rebuilding the relationship between people and nature: The Satoyama initiative. Ecological Research, 25, 891-897. https://doi.org/10.1007/ s11284-010-0745-8.

Tieskens, K. F., Schulpa, C. J. E., Leversb, C., Lieskovsky, J., Kuemmerleb, T., Plieninger, T., et al. (2017). Characterizing European cultural landscapes: Accounting for structure, management intensity and value of agricultural and forest landscapes. Land Use Policy, 62, 29-39. https://doi.org/10.1016/j. landusepol.2016.12.001.

UNESCO. (2008). Operational guidelines for the implementation of the World Heritage Convention. Intergovernmental Committee for The Protection of the World Cultural and Natural Heritage. place de Fontenoy, France: UNESCO World Heritage Centre 7.

UNESCO. (2015). Operational Guidelines for the implementation of the World Heritage Convention. Paris: Intergovernmental Committee for The Protection of The World Cultural and Natural Heritage, UNESCO World Heritage Centre 7.

UNU-IAS, \& IR3S/UTIAS. (2016). In Home garden agroforestry practices in the Gedeo zone, Ethiopia: Asustainable land management system for socio-ecological benefit. Socioecological production landscapes and seascapes (SEPLS) in Africa (pp. 28-36). Tokyo: United Nations University Institute for the Advanced Study of Sustainability.

UNU-IAS, \& IGES. (2016). Mainstreaming concepts and approaches of socio-ecological production landscapes and seascapes into policy and decision-making (Satoyama initiative thematic review vol. 2). Tokyo: United Nations University Institute for the Advanced Study of Sustainability.

Verburg, P. H., van Berkel, D. B., van Doorn, A. M., van Eupen, M., \& van den Heiligenberg Harm, A. R. M. (2010). Trajectories of land use change in Europe: A model-based exploration of rural futures. Landscape Ecology, 25, 217-232. https:// doi.org/10.1007/s10980-009-9347-7.

Vos, W., \& Meekes, H. (1999). Trends in European cultural landscape development: perspectives for a sustainable future. Landsc Urban Planning, 46(1-3), 3-14. https:// doi.org/10.1016/S0169-2046(99)00043-2.

Warnock, S., \& Griffiths, G. (2014). Landscape characterization: The living landscapes approach in the UK. Landscape Research, 40(3), 261-278. https://doi.org/10.1080/ 01426397.2013.870541.

Watson, E. E. (2009). Living terraces in Ethiopia: Konso landscape, culture and development. Eastern Africa Series (p. 256). Boydell \& Brewer, James Currey an Imprint of Boydell \& Brewer.

Wiggering, H., Müller, K., Werner, A., \& Helming, K. (2003). The concept of multifunctionality in sustainable land development. In K. Helming, \& H. Wiggering (Eds.), Sustainable development of multifunctional landscapes. Berlin, Heidelberg: Springer. https://doi.org/10.1007/978-3-662-05240-2_1.

Yirga, D., Abera, M., Kebede, G., \& Kifle, S. (2012). The Gurage and its cultural values. Gurage Zone Ethiopia (Amharic Version): The Gurage Culture, Tourism and Communication Office.

Zanella, L., Sousa, C. H. R., Souza, C. G., Carvalho, L. M. T., \& Borém, R. A. T. (2012). A comparison of visual interpretation and object-based image analysis for deriving landscape metrics. In Proceedings of the 4th GEOBIA, Rio de Janeiro - Brazil (p. 509).

Zewde, B. (1972). The Aymallal Gurage in the nineteenth century: A political history. Trans-African Journal of History, 2(2), 55-68. https://www.jstor.org/stable/ 24520215. 